\title{
The Association between the Serum Uric Acid Level and Hypertension in Middle-Aged and Elderly Adults
}

\author{
Xianpeng Xu $\mathbb{D}^{1},{ }^{1}$ Jinke Huang $\mathbb{D}^{2},{ }^{2}$ Simin Wu $\mathbb{D}^{2},{ }^{2}$ Qingjie Ji $\mathbb{D}^{1},{ }^{1}$ Xuguang Guo $\mathbb{D},{ }^{3}$ \\ and Yong Huang $\mathbb{D}^{4,5}$ \\ ${ }^{1}$ Department of Acupuncture and Moxibustion, Quzhou Hospital of Traditional Chinese Medicine, Quzhou, China \\ ${ }^{2}$ The Second Clinical Medical School, Guangzhou University of Chinese Medicine, Guangzhou, China \\ ${ }^{3}$ Department of Clinical Laboratory Medicine, The Third Affiliated Hospital of Guangzhou Medical University, \\ Guangzhou 510150, China \\ ${ }^{4}$ School of Traditional Chinese Medicine, Southern Medical University, Guangzhou, China \\ ${ }^{5}$ Department of Acupuncture and Moxibustion, Nanfang Hospital, Southern Medical University, Guangzhou, China
}

Correspondence should be addressed to Yong Huang; nanfanglihuang@163.com

Received 29 May 2021; Accepted 11 October 2021; Published 26 October 2021

Academic Editor: Victor Garcia

Copyright (C) 2021 Xianpeng Xu et al. This is an open access article distributed under the Creative Commons Attribution License, which permits unrestricted use, distribution, and reproduction in any medium, provided the original work is properly cited.

\begin{abstract}
Background. Studies on serum uric acid (sUA) levels and hypertension (HTN) are controversial. To investigate the association between the sUA level and the incident of HTN in middle-aged and elderly adults, we performed this study. Methods. 6399 participants aged $\geq 40$ years from the National Health and Nutrition Examination Survey (NHANES) were included. Weighted multiple logistic regression analysis was carried out to evaluate the relationship between the sUA level and the incident of HTN, exploring the potential nonlinear relationship using the fitted smoothing curves. If nonlinearity was observed, the inflection point was further calculated by a recursive algorithm. Results. A positive relationship between the sUA level and the incident of HTN was found. However, it may differ in different race groups, nor between male and female. Moreover, the association between the sUA level and the incident of HTN followed a U-shaped curve in male (turning point: sUA $4.1 \mathrm{mg} / \mathrm{dL}$ ) and Whites (turning point: sUA $7.9 \mathrm{mg} / \mathrm{dL}$ ). Conclusions. The results revealed that the sUA level is positively correlated with the incident of HTN, in middle-aged and elderly adults. However, it followed a U-shaped curve in males and Whites.
\end{abstract}

\section{Introduction}

Worldwide, hypertension (HTN) is both a disease and a major modifiable risk factor for all-cause morbidity and mortality [1]. It is reported that more than 100 million people are currently affected by HTN and it is expected that more than $29.2 \%$ of adults will suffer from this disease in 2025 [2]. HTN rarely causes symptoms in its early stages, so less than half of HTN patients value their condition, although early diagnosis and treatment of HTN are essential [1]. Hence, a better understanding of modifiable risk factors for HTN is useful for early detection and prevention of HTN, which could help reduce this disease and its associated complications.

As a natural component of blood, serum uric acid (sUA) is the ultimate product of purine metabolism [3]. In recent years, epidemiological data have found inconsistent conflicting results on the association between sUA and HTN [4]. Elevated sUA reduces susceptibility to nitric oxide, endothelial dysfunction, and damage to the renal angiotensin aldosterone system, which eventually causes blood vessels to constrict $[5,6]$. However, there are many different views on this issue due to the complex factors associated with sUA and HTN and controversial findings have been reported. Specifically, while an elevated sUA level was associated with an increased risk of developing HTN [4, 7-11], most other observational or Mendelian randomized studies did not support these associations [12-14]. Therefore, the causal relationship between sUA levels and HTN remains to be examined. To evaluate the association between them in middle-aged and elderly adults, data from the United 


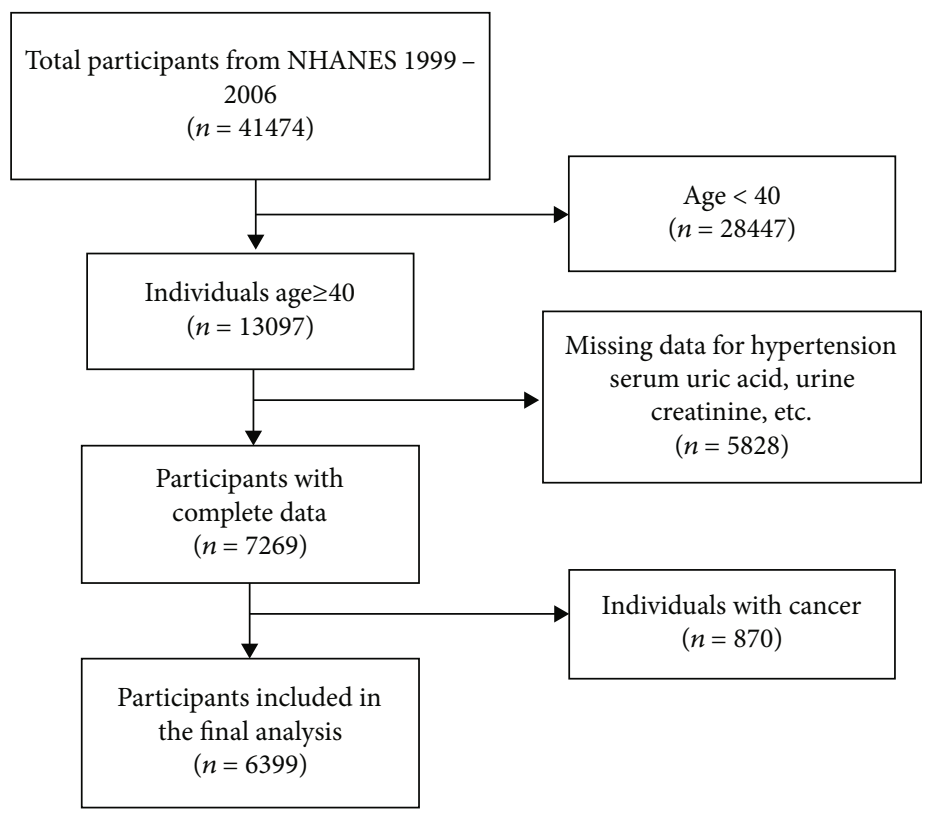

FIGURE 1: Sample screening flow chart.

States (US) National Health Nutrition and Examination Survey (NHANES) were used to perform this study.

\section{Methods}

2.1. Study Population. The NHANES database collected health examination data from the noninstitutionalized US population [15]. The NHANES study was approved by the Institutional Review Board of the National Center for Health Statistics. 41474 participants were identified from NHANES 2001-2006, and 35075 participants without complete measurement data or the health status did not meet the inclusion criteria were excluded. Finally, 6399 participants with completed data were analyzed in this study (Figure 1).

2.2. Study Variables. The exposure variable was serum sUA. From 1999 to 2001, the sUA levels were measured using Roche Hitachi Model 917 or 704 Multichannel Analyzer, while the Beckman Synchron LX20 was used since 2002 [16].

The outcome of interest was blood pressure status measuring by trained research physicians. HTN was defined as self-reported HTN. Subjects with systolic BP $\geq 130 \mathrm{mmHg}$ or diastolic $\mathrm{BP} \geq 80 \mathrm{mmHg}$ were considered to have HTN [17].

Additionally, other covariates included age, sex, race, income-poverty ratio, educational level, body mass index (BMI), diabetes mellitus status, physical activity, smoking behavior, alcohol consumption, total cholesterol, serum homocysteine (Hcy), urine creatinine, blood urea nitrogen, and serum calcium.

2.3. Statistical Analysis. R (version 3.4.3) and EmpowerStats (X\&Y Solutions, Boston, MA) were applied to performed statistical analysis. Sample weights were calculated considering all estimates from NHANES. Categorical variables were presented using frequencies or percentages, and continuous variables were mean \pm standard deviation. After adjustment for potential confounders, weighted multivariate linear regression models and smooth curve fitting were performed to evaluate the association of sUA levels with HTN incidence. The weighted linear regression model was performed to calculate the difference of continuous variables; for categorical variables, the weighted chi-square test was used. $P<0.05$ was considered statistically significant.

\section{Results}

3.1. Characteristics of Participants. Characteristics of the included subjects were subdivided followed by the sUA quartiles (Q1: 1.5-4.3 mg/dL; Q2: 4.4-5.2 mg/dL; Q3: 5.3$6.3 \mathrm{mg} / \mathrm{dL}$; and $\mathrm{Q} 4: 6.4-13.7 \mathrm{mg} / \mathrm{dL}$ ), and the medians of these chosen quartiles were $3.8 \mathrm{mg} / \mathrm{dL}, 4.8 \mathrm{mg} / \mathrm{dL}, 5.8 \mathrm{mg} / \mathrm{dL}$, and $7.2 \mathrm{mg} / \mathrm{dL}$. Among different groups of sUA, significant differences in baseline characteristics were observed, with the exception of education, income, physical activity, and total cholesterol (Table 1).

3.2. Association between sUA and HTN. Three models were developed: model 1, unadjusted; model 2, sex, age, and race were adjusted; and model 3 , adjusted for covariates presented in Table 1 . In all models, sUA was positively associated to the incidence of HTN (Table 2, Figure 2). After converting sUA from a continuous variable to a categorical variable (quartiles), the odds ratios (ORs) for the association of sUA and HTN in the other three groups were 1.25, 1.53, and 2.11 , respectively, with the lowest quartile as the reference. When stratified by sex, a positive association was found between sUA and HTN. When stratified by race, sUA was positively related to the incidence of HTN in Blacks, Whites, and Mexican American, but not in other races (OR $1.14(0.97,1.33))$. 
TABLE 1: Characteristics of participants.

\begin{tabular}{|c|c|c|c|c|c|c|}
\hline Serum uric acid & Total & Q1 & Q2 & Q3 & Q4 & $P$ value \\
\hline Age & $59.56 \pm 13.05$ & $56.66 \pm 12.66$ & $59.63 \pm 12.75$ & $60.31 \pm 13.04$ & $61.15 \pm 13.25$ & $<0.0001$ \\
\hline Sex $(\%)$ & & & & & & $<0.0001$ \\
\hline Female & 50.57 & 78.88 & 59.93 & 39.12 & 30.15 & \\
\hline Male & 40.43 & 21.12 & 40.07 & 60.88 & 69.85 & \\
\hline Race (\%) & & & & & & $<0.0001$ \\
\hline Non-Hispanic White & 50.16 & 46.29 & 49.68 & 51.78 & 52.19 & \\
\hline Non-Hispanic Black & 19.24 & 17.14 & 15.37 & 20.38 & 23.29 & \\
\hline Mexican American & 23.64 & 29.57 & 27.23 & 21.12 & 18.02 & \\
\hline Other race & 6.96 & 7.00 & 7.71 & 6.71 & 6.50 & \\
\hline Educational level (\%) & & & & & & 0.4106 \\
\hline Less than high school & 35.67 & 33.57 & 36.56 & 36.67 & 35.63 & \\
\hline High school & 22.30 & 21.83 & 22.32 & 21.96 & 23.01 & \\
\hline $\begin{array}{l}\text { College graduate } \\
\text { or above }\end{array}$ & 42.03 & 44.60 & 41.12 & 41.37 & 41.36 & \\
\hline $\begin{array}{l}\text { Body mass index } \\
\left(\mathrm{kg} / \mathrm{m}^{2}\right)\end{array}$ & $28.92 \pm 6.05$ & $26.76 \pm 5.40$ & $28.37 \pm 5.86$ & $29.48 \pm 6.21$ & $30.63 \pm 5.94$ & $<0.0001$ \\
\hline Income poverty ratio & $2.73 \pm 1.53$ & $2.69 \pm 1.55$ & $2.71 \pm 1.52$ & $2.74 \pm 1.53$ & $2.79 \pm 1.52$ & 0.2925 \\
\hline Any hypertension (\%) & & & & & & $<0.0001$ \\
\hline No & 57.19 & 68.57 & 60.89 & 56.58 & 45.02 & \\
\hline Yes & 42.81 & 31.43 & 39.11 & 43.42 & 54.98 & \\
\hline $\begin{array}{l}\text { Diabetes mellitus } \\
\text { status (\%) }\end{array}$ & & & & & & $<0.0001$ \\
\hline No & 82.54 & 84.12 & 84.28 & 83.77 & 78.44 & \\
\hline Yes & 17.46 & 15.88 & 15.72 & 16.23 & 21.56 & \\
\hline Physical activity (100\%) & & & & & & 0.0054 \\
\hline 0 & 27.32 & 27.31 & 26.74 & 27.05 & 28.37 & \\
\hline 1 & 27.50 & 26.05 & 29.81 & 28.21 & 25.39 & \\
\hline 2 & 17.81 & 20.58 & 15.19 & 17.38 & 18.54 & \\
\hline 3 & 27.37 & 26.06 & 28.26 & 27.37 & 27.69 & \\
\hline Smoking behavior (\%) & & & & & & $<0.0001$ \\
\hline No & 48.32 & 57.60 & 49.58 & 47.01 & 40.78 & \\
\hline Yes & 51.68 & 42.40 & 50.42 & 52.99 & 59.22 & \\
\hline $\begin{array}{l}\text { Alcohol consumption } \\
(\%)\end{array}$ & & & & & & $<0.0001$ \\
\hline No & 73.69 & 80.85 & 74.91 & 74.08 & 66.24 & \\
\hline Yes & 26.31 & 19.15 & 25.09 & 25.92 & 33.76 & \\
\hline $\begin{array}{l}\text { Total cholesterol } \\
(\mathrm{mg} / \mathrm{dL})\end{array}$ & $210.16 \pm 41.25$ & $209.02 \pm 39.64$ & $211.21 \pm 41.11$ & $208.49 \pm 40.60$ & $211.85 \pm 43.21$ & 0.0591 \\
\hline Serum Hcy $(\mu \mathrm{mol} / \mathrm{L})$ & $9.75 \pm 6.09$ & $8.50 \pm 7.26$ & $8.96 \pm 4.55$ & $9.83 \pm 3.89$ & $11.43 \pm 7.49$ & $<0.0001$ \\
\hline $\begin{array}{l}\text { Urine creatinine } \\
(\mu \mathrm{mol} / \mathrm{L})\end{array}$ & $10650.25 \pm 6824.07$ & $8936.80 \pm 6387.75$ & $9917.44 \pm 6463.99$ & $11454.07 \pm 6570.79$ & $11921.86 \pm 7339.25$ & $<0.0001$ \\
\hline $\begin{array}{l}\text { Blood urea nitrogen } \\
(\mathrm{mg} / \mathrm{dL})\end{array}$ & $14.93 \pm 6.31$ & $13.05 \pm 4.61$ & $13.86 \pm 4.77$ & $15.06 \pm 5.82$ & $17.33 \pm 8.17$ & $<0.0001$ \\
\hline Total calcium (mg/dL) & $9.44 \pm 0.40$ & $9.36 \pm 0.39$ & $9.45 \pm 0.41$ & $9.48 \pm 0.40$ & $9.47 \pm 0.40$ & $<0.0001$ \\
\hline
\end{tabular}

Mean \pm SD for continuous variables: $P$ value was calculated by the weighted linear regression model. $\%$ for categorical variables: $P$ value was calculated by the weighted chi-square test.

For subgroup analysis (Table 3), a significant association between the sUA level and incidence of HTN was found in all female except those of other races ( $P$ for trend $=0.1154$ ). In the male population, the sUA level was significantly associated with the incidence of HTN ( $P$ for trend $>0.05$ ), except for Blacks and other race subjects. We tried to use generalized additive models and smooth curve fittings to identify the nonlinear relationship stratified by sex and race. 
TABLE 2: Association between sUA and HTN.

\begin{tabular}{lccr}
\hline & Model 1, OR $(95 \%$ CI $)$ & Model 2, OR $(95 \%$ CI $)$ & Model 3, OR (95\% CI) \\
\hline Serum uric acid & $1.32(1.27,1.37)$ & $1.36(1.31,1.42)$ & $1.22(1.17,1.28)$ \\
Serum uric acid categories & Reference & Reference & Reference \\
Q1 & $1.46(1.25,1.70)$ & $1.48(1.26,1.73)$ & $1.25(1.06,1.47)$ \\
Q2 & $1.85(1.60,2.15)$ & $2.05(1.75,2.40)$ & $1.53(1.29,1.82)$ \\
Q3 & $2.84(2.45,3.29)$ & $3.22(2.72,3.80)$ & $2.11(1.76,2.54)$ \\
Q4 & & & $1.30(1.21,1.39)$ \\
Stratified by sex & $1.63(1.53,1.73)$ & $1.49(1.40,1.58)$ & $1.17(1.10,1.24)$ \\
$\quad$ Female & $1.28(1.21,1.35)$ & $1.26(1.19,1.33)$ & $1.29(1.21,1.38)$ \\
$\quad$ Male & $1.40(1.33,1.48)$ & & $1.18(1.39,1.57)$ \\
Stratified by race & $1.26(1.17,1.37)$ & $1.31(1.20,1.43)$ & $1.07,1.31)$ \\
$\quad$ Non-Hispanic White & $1.18(1.09,1.27)$ & $1.22(1.12,1.33)$ & $1.18(1.07,1.30)$ \\
$\quad$ Non-Hispanic Black & $1.24(1.09,1.41)$ & $1.27(1.11,1.47)$ & $1.14(0.97,1.33)$ \\
$\quad$ Mexican American & & & \\
$\quad$ Other race & & & \\
\hline
\end{tabular}

Model 1: no covariates were adjusted; model 2: age, sex, and race were adjusted; model 3: age, sex, race, income-poverty ratio, physical activity, smoking behavior, alcohol consumption, diabetes mellitus status, body mass index, total cholesterol, serum homocysteine, urine creatinine, blood urea nitrogen, and total calcium were adjusted.

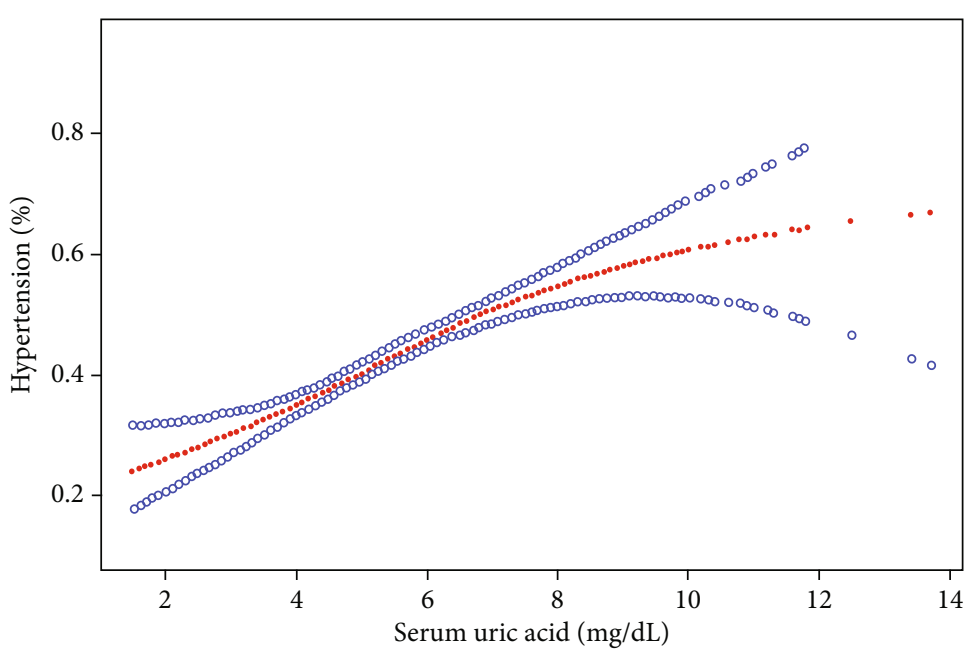

Figure 2: Association between sUA and HTN. The area between two blue dotted lines is expressed as a 95\% CI. Age, sex, race, incomepoverty ratio, physical activity, smoking behavior, alcohol consumption, diabetes mellitus status, body mass index, total cholesterol, serum homocysteine, urine creatinine, blood urea nitrogen, and total calcium were adjusted.

As shown in Figure 2, there was an overall linear relationship between sUA and HTN incidence but subgroup analysis showed a nonlinear relationship between sUA and the HTN incidence in male and Whites (Figures 3 and 4). The incidence of HTN did not increased with sUA up to the turning point in male (turning point: sUA $4.1 \mathrm{mg} / \mathrm{dL}$ ) (Table 4). Likewise, there were turning points in Whites (turning point: sUA $7.9 \mathrm{mg} / \mathrm{dL}$ ) (Table 4). Taken together, the association between sUA and the incidence of HTN in male and Whites followed an inverted U-shaped curve.

\section{Discussion}

Epidemiologic data suggested an association between sUA and HTN. However, due to the complex factors associated with sUA and HTN, there were many different views on this issue and controversial results had been reported in this limited body of evidence. Thus, we aimed to investigate whether sUA was independently associated with the incidence of HTN, using a large and nationally representative sample of middle-aged and elderly adults in the US. Study findings showed that the incidence of HTN was statistically significantly higher with the increasing baseline levels of sUA, indicating that the higher baseline sUA level is an independent risk factor for HTN. However, the association between the sUA level and the incident of HTN followed a U-shaped curve for male (turning point: sUA $4.1 \mathrm{mg} / \mathrm{dL}$ ) and Whites (turning point: sUA $7.9 \mathrm{mg} / \mathrm{dL}$ ).

Previous studies have found an association between sUA and HTN. A cohort study performed in the US showed a 
TABLE 3: Subgroup analysis stratified by race and sex.

\begin{tabular}{|c|c|c|c|c|}
\hline \multirow{2}{*}{ Quartiles of serum uric acid } & \multicolumn{4}{|c|}{ Incidence of HTN OR (95\% CI) } \\
\hline & Whites & Blacks & Mexican Americans & Other races \\
\hline \multicolumn{5}{|l|}{ Female } \\
\hline Lowest quartiles & Reference & Reference & Reference & Reference \\
\hline 2nd & $1.38(1.04,1.85)$ & $1.25(0.78,1.99)$ & $1.58(1.07,2.33)$ & $0.85(0.41,1.76)$ \\
\hline $3 \mathrm{rd}$ & $1.67(1.21,2.31)$ & $1.56(0.94,2.61) 0.0864$ & $1.64(1.02,2.64) 0.0417$ & $1.71(0.74,3.97) 0.2090$ \\
\hline 4 th & $2.48(1.68,3.67)$ & $2.04(1.14,3.63)$ & $2.14(1.13,4.05)$ & $1.89(0.75,4.79)$ \\
\hline$P$ for trend & $<0.001$ & 0.0114 & 0.0074 & 0.1154 \\
\hline \multicolumn{5}{|l|}{ Male } \\
\hline Lowest quartiles & Reference & Reference & Reference & Reference \\
\hline 2nd & $1.59(0.96,2.65)$ & $0.54(0.24,1.18)$ & $0.99(0.54,1.79)$ & $0.36(0.10,1.34)$ \\
\hline $3 \mathrm{rd}$ & $1.65(1.02,2.67)$ & $1.03(0.53,2.03)$ & $1.56(0.88,2.75)$ & $0.41(0.11,1.47)$ \\
\hline 4 th & $2.61(1.60,4.24)$ & $1.18(0.61,2.28)$ & $1.59(0.87,2.89)$ & $0.69(0.19,2.48)$ \\
\hline$P$ for trend & $<0.001$ & 0.1407 & 0.0396 & 0.5788 \\
\hline
\end{tabular}

Age, sex, race, income-poverty ratio, physical activity, smoking behavior, alcohol consumption, diabetes mellitus status, body mass index, total cholesterol, serum homocysteine, urine creatinine, blood urea nitrogen, and total calcium were adjusted.

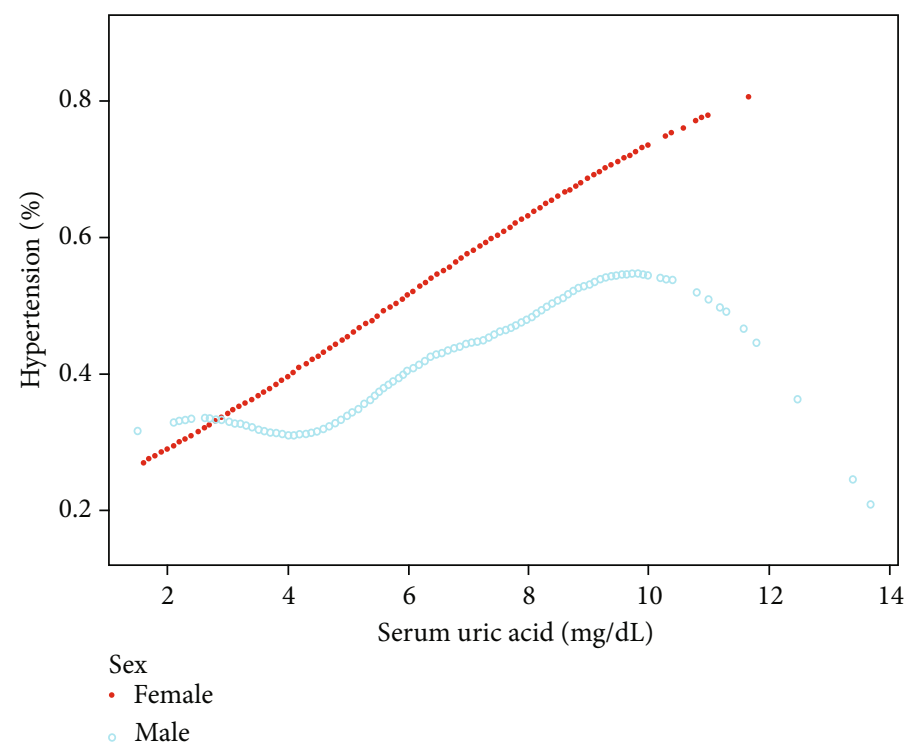

Figure 3: Association between sUA and HTN stratified by sex. Age, race, income-poverty ratio, physical activity, smoking behavior, alcohol consumption, diabetes mellitus status, body mass index, total cholesterol, serum homocysteine, urine creatinine, blood urea nitrogen, and total calcium were adjusted.

dose-dependent increase in the relative risk of HTN with increasing quartiles of sUA [18]. A meta-analysis enrolled 18 prospective studies with a total of 55607 participants concluded that a high level of sUA was an independent risk factor for HTN, and the risk ratio for incident HTN was 1.13 with each increase of $1 \mathrm{mg} / \mathrm{dL}$ in the sUA level after adjusting for potential confounding factors, which was similar to that in our study [19]. Mechanisms underlying the relationship between sUA and HTN include a reduction in endothelial nitric oxide, the activation of the renin-angiotensin system, and renal microvascular disease caused by smooth muscle cell proliferation, inflammation, and local reninangiotensin system activation [8]. However, another observational study and the Mendelian randomized studies did not support a causal association between sUA and HTN [12-14]. These conflicting conclusions may be attributed to differences in demographic characteristics, study design, study size, controlling for confounding factors, etc.

The first interesting finding was that the relationship between sUA and the incidence of HTN in male followed an inverted U-shaped curve. For male, the incident of HTN did not increase with increasing sUA until the turning point $(4.1 \mathrm{mg} / \mathrm{dL})$. A cross-sectional study of 85286 Japanese workers found similar results [20]. A significant relationship was found between sUA and HTN when sUA was $\geq 5.3 \mathrm{mg} / \mathrm{dL}$ in male. It was observed that there was a different relationship between sUA and HNT in people of different genders [19]. Previous studies [21] have shown that 


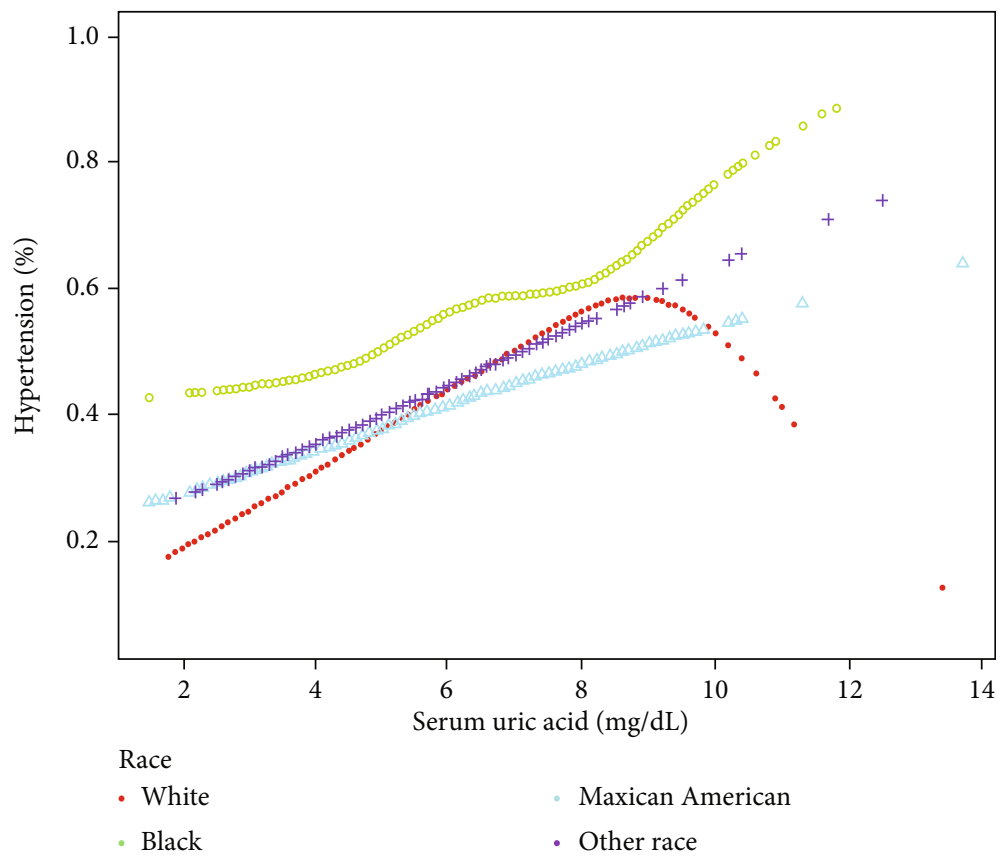

Figure 4: Association between sUA and HTN, stratified by race. Age, sex, income-poverty ratio, physical activity, smoking behavior, alcohol consumption, diabetes mellitus status, body mass index, total cholesterol, serum homocysteine, urine creatinine, blood urea nitrogen, and total calcium were adjusted.

TABLE 4: Threshold effect analysis.

\begin{tabular}{lc}
\hline Serum uric acid & Adjusted OR $(95 \%$ CI $), P$ value \\
\hline Male & \\
$\quad$ Serum uric acid $<4.1(\mathrm{mg} / \mathrm{dL})$ & $0.79(0.47,1.31) 0.3574$ \\
$\quad$ Serum uric acid $>4.1(\mathrm{mg} / \mathrm{dL})$ & $1.19(1.11,1.27)<0.0001$ \\
White & $1.34(1.24,1.45)<0.0001$ \\
$\quad$ Serum uric acid $<7.9(\mathrm{mg} / \mathrm{dL})$ & $0.85(0.61,1.18) 0.3195$ \\
$\quad$ Serum uric acid $>7.9(\mathrm{mg} / \mathrm{dL})$ & \\
\hline
\end{tabular}

Age, sex, race, income-poverty ratio, physical activity, smoking behavior, alcohol consumption, diabetes mellitus status, body mass index, total cholesterol, serum homocysteine, urine creatinine, blood urea nitrogen, and total calcium were adjusted.

men have higher circulating levels of sUA than women. This gender difference may be explained as a result of the inhibitory effect of estradiol on sUA, as estradiol inhibits the isolated urate-producing enzyme and also decreases circulating uric acid in a pharmacological manner [22, 23]. Moreover, sex hormones have a potential impact on the relationship between sUA and HTN, as sUA has been found to be associated with the internal carotid artery resistance index and pulse wave velocity in female, but this phenomenon has not been observed in male $[24,25]$. Thus, to understand the mechanisms underlying sex differences, further studies on the role of sex hormones are needed. Gender differences should also be taken into account in the prevention of hyperuricemic complications $[4,26]$.

The second interesting finding was that the relationship between sUA and the incidence of HTN in Whites followed an inverted U-shaped curve. The incident of HTN decreased when sUA levels reached $7.9 \mathrm{mg} / \mathrm{dL}$. So far, evidence linking sUA and HTN in different races was very limited. Although there were studies on the association between sUA and HTN in Japan and China, however, these studies were conducted separately for their countries, where the races were all Yellows and ethnic factors were not taken into account at the study design stage, which made it impossible to compare the association between sUA and HTN among different races. To our knowledge, this was the first study to report the association between sUA and HTN in different races in US adults. Although the association between sUA and HTN in Whites following a U-shaped curve was an interesting finding, this phenomenon cannot be explained due to the lack of existing evidence. Thus, future prospective studies with large samples for different races are needed for further validation. Polymorphisms in the regulator of G-protein signaling 2 gene have been reported to be associated with HTN in Blacks but not in Whites [27].

Therefore, we speculated that genetic differences may be a potential explanation for the presence of a U-shaped curve in Whites, which was different from other races. However, further studies on the role of genes are needed to understand the emphasized mechanism of ethnic differences.

4.1. Limitations. In this study, the representative samples of the multiracial population were included to better generalize of the US population; the large sample size enables us to conduct further subgroup analyses for sensitivity test and to adjust many potential confounding factors. However, limitations must also be acknowledged. First, due to the cross-sectional design of this study, the causal relationship between sUA and HTN cannot be elucidated. Second, the 
diagnosis of HTN was based on the patients' self-report, which may lead to the risk of bias. Third, participants with cancer were excluded because these special populations have a great influence on sUA and HTN. Thus, the conclusions of this study cannot be applied to these patients. Fourth, the bias caused by other potential confounding factors that did not adjust in this study is not excluded.

\section{Conclusion}

In conclusion, this cross-sectional study suggested that the sUA level positively correlated with the incident of HTN, in middle-aged and elderly adults. However, the association the between sUA level and the incident of HTN in males and Whites followed a U-shaped curve.

$\begin{array}{ll}\text { Abbreviations } \\ \text { sUA: } & \text { Serum uric acid } \\ \text { HTN: } & \text { Hypertension } \\ \text { NHANES: } & \text { National Health and Nutrition Examination } \\ & \text { Survey } \\ \text { US: } & \text { United States } \\ \text { BMI: } & \text { Body mass index } \\ \text { Hcy: } & \text { Homocysteine } \\ \text { OR: } & \text { Odds ratio. }\end{array}$

\section{Data Availability}

The survey data are publicly available on the Internet for data users and researchers throughout the world http:// www.cdc.gov/nchs/nhanes/.

\section{Conflicts of Interest}

The authors declare that there is no conflict of interest.

\section{Authors' Contributions}

Xianpeng Xu contributed to the study design and writing of the manuscript. Jinke Huang, Simin Wu, Qingjie Ji, and Xuguang Guo contributed to data collection and analysis. Yong Huang provided guidance on the study methodology. All authors read, critically reviewed, and approved the final manuscript as submitted.

\section{Acknowledgments}

The authors thank all investigators and participants involved the NHANES study for their valuable contributions.

\section{References}

[1] S. Oparil, M. C. Acelajado, G. L. Bakris et al., "Hypertension," Nature Reviews. Disease Primers, vol. 4, no. 1, 2018.

[2] P. M. Kearney, M. Whelton, K. Reynolds, P. Muntner, P. K. Whelton, and J. He, "Global burden of hypertension: analysis of worldwide data," The Lancet, vol. 365, no. 9455, pp. 217223, 2005.
[3] G. K. Glantzounis, E. C. Tsimoyiannis, A. M. Kappas, and D. A. Galaris, "Uric acid and oxidative stress," Current Pharmaceutical Design, vol. 11, no. 32, pp. 4145-4151, 2005.

[4] L. F. Cui, H. J. Shi, S. L. Wu et al., "Association of serum uric acid and risk of hypertension in adults: a prospective study of Kailuan Corporation cohort," Clinical Rheumatology, vol. 36, no. 5, pp. 1103-1110, 2017.

[5] M. Mazzali, J. Hughes, Y. G. Kim et al., "Elevated uric acid increases blood pressure in the rat by a novel crystalindependent mechanism," Hypertension, vol. 38, no. 5, pp. 1101-1106, 2001.

[6] M. Mazzali, M. Kanbay, M. S. Segal et al., "Uric acid and hypertension: cause or Effect?," Current Rheumatology Reports, vol. 12, no. 2, pp. 108-117, 2010.

[7] X. Niu, J. Chen, J. Wang et al., "Association between the uric acid and hypertension in community-based Chinese population: stratified analysis based on body mass index and age," Journal of Thrombosis and Thrombolysis, vol. 51, no. 4, pp. 1113-1119, 2021.

[8] I. Miyabayashi, S. Mori, A. Satoh et al., "Uric acid and prevalence of hypertension in a general population of Japanese: ISSA-CKD study," Journal of Clinical Medical Research, vol. 12, no. 7, pp. 431-435, 2020.

[9] M. Kuwabara, K. Niwa, Y. Nishi et al., "Relationship between serum uric acid levels and hypertension among Japanese individuals not treated for hyperuricemia and hypertension," Hypertension Research, vol. 37, no. 8, pp. 785-789, 2014.

[10] M. Kuwabara, K. Niwa, I. Hisatome et al., "Asymptomatic hyperuricemia without comorbidities predicts cardiometabolic Diseases," Hypertension, vol. 69, no. 6, pp. 1036-1044, 2017.

[11] M. Kuwabara, I. Hisatome, K. Niwa et al., "Uric acid is a strong risk marker for developing hypertension from prehypertension: a 5-year Japanese cohort study," Hypertension, vol. 71, no. 1, pp. 78-86, 2018.

[12] S. Sedaghat, R. Pazoki, A. G. Uitterlinden et al., “Association of uric acid genetic risk score with blood pressure: the Rotterdam study," Hypertension, vol. 64, no. 5, pp. 1061-1066, 2014.

[13] J. Wang, T. Qin, J. Chen et al., "Hyperuricemia and risk of incident hypertension: a Systematic review and meta-analysis of observational studies," PLoS One, vol. 9, no. 12, article e114259, 2014.

[14] X. Li, X. Meng, M. Timofeeva et al., "Serum uric acid levels and multiple health outcomes: umbrella review of evidence from observational studies, randomised controlled trials, and Mendelian randomisation studies," BMJ, vol. 357, p. j2376, 2017.

[15] L. R. Curtin, L. K. Mohadjer, S. M. Dohrmann et al., “The National Health and Nutrition Examination Survey: sample design, 1999-2006," Vital and Health Statistics. Series 2. Data Evaluation and Methods Research, no. 155, pp. 1-39, 2012.

[16] G. Zipf, M. Chiappa, K. S. Porter, Y. Ostchega, B. G. Lewis, and J. Dostal, "National Health and Nutrition Examination Survey: plan and operations, 1999-2010," Vital Health Statistics Series 1 Programs Collection Procedures, no. 56, pp. 1-37, 2013.

[17] P. K. Whelton, R. M. Carey, W. S. Aronow et al., "2017 ACC/AHA/AAPA/ABC/ACPM/AGS/APhA/ASH/ASPC/N$\mathrm{MA} / \mathrm{PCNA}$ guideline for the prevention, detection, evaluation, and management of high blood pressure in adults: Executive Summary: a report of the American College of Cardiology/American Heart Association Task Force on Clinical Practice Guidelines," Hypertension, vol. 71, no. 6, pp. 1269-1324, 2018. 
[18] A. Shankar, R. Klein, B. E. Klein, and F. J. Nieto, "The association between serum uric acid level and long-term incidence of hypertension: population-based cohort study," Journal of Human Hypertension, vol. 20, no. 12, pp. 937-945, 2006.

[19] P. C. Grayson, S. Y. Kim, M. LaValley, and H. K. Choi, "Hyperuricemia and incident hypertension: a systematic review and meta-analysis," Arthritis Care \& Research, vol. 63, no. 1, pp. 102-110, 2011.

[20] H. Yokokawa, H. Fukuda, A. Suzuki et al., "Association between serum uric acid levels/hyperuricemia and hypertension among 85,286 Japanese workers," The Journal of Clinical Hypertension, vol. 18, no. 1, pp. 53-59, 2016.

[21] Y. Wang and F. J. Charchar, "Establishment of sex difference in circulating uric acid is associated with higher testosterone and lower sex hormone-binding globulin in adolescent boys," Scientific Reports, vol. 11, no. 1, p. 17323, 2021.

[22] K. Huh, U.-S. Shin, J.-W. Choi, and S.-I. Lee, "Effect of sex hormones on lipid peroxidation in rat liver," Archives of Pharmacal Research, vol. 17, no. 2, pp. 109-114, 1994.

[23] R. Yahyaoui, I. Esteva, J. J. Haro-Mora et al., "Effect of longterm administration of cross-sex hormone therapy on serum and urinary uric acid in transsexual persons," The Journal of Clinical Endocrinology \& Metabolism, vol. 93, no. 6, pp. 2230-2233, 2008.

[24] M. A. Gomez-Marcos, J. I. Recio-Rodriguez, M. C. PatinoAlonso et al., "Relationship between uric acid and vascular structure and function in hypertensive patients and sexrelated differences," American Journal of Hypertension, vol. 26, pp. 599-607, 2013.

[25] J.-I. Fang, J.-S. Wu, Y.-C. Yang, R.-H. Wang, F.-H. Lu, and C.J. Chang, "High uric acid level associated with increased arterial stiffness in apparently healthy women," Atherosclerosis, vol. 236, no. 2, pp. 389-393, 2014.

[26] A. Samimi, S. Ramesh, T. C. Turin et al., "Serum uric acid level, blood pressure, and vascular angiotensin II responsiveness in healthy men and women," Physiological Reports, vol. 2, no. 12, article e12235, 2014.

[27] I. N. Hahntow, G. Mairuhu, I. G. van Valkengoed et al., "Are RGS2 gene polymorphisms associated with high blood pressure in an ethnicity- and gender-specific manner?," American Journal of Hypertension, vol. 22, no. 1, pp. 80-86, 2009. 